\title{
CISTICERCOSE MEDULAR
}

RELATO DE DOIS CASOS, REVISÃo DA LITERATURA B COMENTARIOS SOBRE A PATOGENIA

\author{
NEVAIR R. GALLANI * - HÉLDER J. L. ZAMBELLI ** \\ A. A. ROTH-VARGAS, *** - CELSO LIMOLI JR.****
}

\begin{abstract}
RESUMO - Relato de dois casos de cisticercose medular, sendo o primeiro referente a uma paciente de 13 anos de idade, apresentando a forma intramedular, e o segundo a uma paciente de 51 anos de idade com cisticercose espinha>l leptomeníngea. Numa revisão de literatura (desde 1856), foram encontrados 95 casos publicados de cisticercose medular somados a dois de nossa casuística, mostrando a freqüência da doença. Dentro da patogenia da forma extramedular, os autores defendem a hipótese da migração dos cisticercos do espaço subaracnófde intracraniano para o espinhal, baseando-se na distribuição topográfica dos parásitos na medula.
\end{abstract}

PALAVRAS-CHAVE: cisticercose, medula espinhal, canal raquidiano, patogenia.

Cysticercosis of the spinal cord: report of two cases, literature review and comments on pathogenesis.

SUMMARY - A case of spinal intramedular cysticercosis in a 13-year-old Brazilian female and a case of spinal leptomeningeal infestation by cysticercosis in a 51 year-old Brazilian female are presented. A review of 95 published cases of medullar cysticercosis since 1856 shows the incidence of this condition. Extramedullar forms are explained by the downward migration of larvae from the cerebral to the spinal subarachnoid space and most larvae are expected to be stopped in the upper portions of the spinal canal due to peculiarities of the anatomy of the spinal cord.

KEY WORDS : cysticercosis, spinal cord, spinal canal, pathogenesis.

Cisticercose é a parasitose mais comum do sistema nervoso, constituindo doença endêmica no Brasil, como em outros países com baixo nível sanitário. Esta parasitose, ocorre no homem por desvio do ciclo de vida da Taenia solium, já que são os suínos os hospedeiros intermediários habituais. Fora do sistema nervoso, outros sítios de infestação incluem o tecido celular subcutâneo, musculatura esquelética, globo ocular e, mais raramente, o coração, pulmões, peritônio, pâncreas, aorta, tiróide, jejuno (submucosa), traqueia, fígado e osso $5,8,29,30$.

Embora frequente na localização encefálica, o encontro do parasito na medula é relativamente raro. Contudo, deve-se lembrar que a real incidência de cisticercose medular, provavelmente, é subestimada devido a dominar a sintomatologia cerebral o quadro clínico e não ser a medula objeto de análise de rotina em necropsias. Dois casos de cisticercose medular são registrados.

Disciplina de Neurocirurgia do Departamento de Neurologia, Faculdade de Ciências Médicas da Universidade Estadual de Campinas (UNICAMP) e Departamento de Neurologia da Santa Casa de Valinhos: * Médico Residente; ** Graduando de Medicina; *** Neurocirurgião, Professor Assistente da UNICAMP; **** Neurocirurgião, Chefe do Departamento de Neurologia dia Santa Casa de Valinhos.

Dr. Antonio Augusto Roth-Vargas - Departamento de Neurologia, Faculdade de Ciências Médicas, UNICAMP - Caixa Postal Cãll - 13081 Campinas SP - Brasil. 


\section{RELATO DOS CASOS}

Caso 1 - RSJ, paciente com 13 anos de idade, do sexo feminino, estudante, com queixa de lombalgia há 6 meses. Nesse período foi submetida a radiografia simples de coluna que se mostrou normal e a tratamento fisioterápico. Evo\&uiu com piora da dor que se irradiou para f)ace posterior de coxas, bilateralmente. Realizaram-se então: tomografia computadorizada (TC) de coluna tóraco-lombar, sem evidenciar anormalidade; estudo eletromiográfico em membros inferiores e musculatura paravertebral, sendo encontradas fibrilações e ondas positivas numerosas, sugerindo ação denervativa recente nos níveis Til, T12 e LI à esquerda (E). Foi realizado ainda novo exame tomográfico entre $T l$ e T8, com injeção de contraste intratecal, sendo considerado normal. Nesta época foi admitida em nosso serviço, apresentando-se com lombalgia intensa, dificuldade à deambulação, reflexos patelares e aquilianos vivos com predomínio à $\mathrm{E}$, paraparesia crural discreta, disestesia em dermátomos de $\mathbf{L} 3$ a $I A$ à $\mathbf{E}$ e dor em faixa nos níveis correspondentes a Til e T12. A mielografia tóraco-lombar mostrou bloqueio completo entre Til e T12; na mesma oportunidade, pela TC era diagnosticada, nesse nível, lesão hipodensa intramedular central comprometendo praticamente todo o diâmetro da medula (Fig 1). Em 19-julho-89 foi submetida a mielotomia posterior segundo a técnica de Kempe, com extrusão de uma vesícula que, posteriormente, o exame anátomo-patológico confirmou tratar-se de um cisticerco.

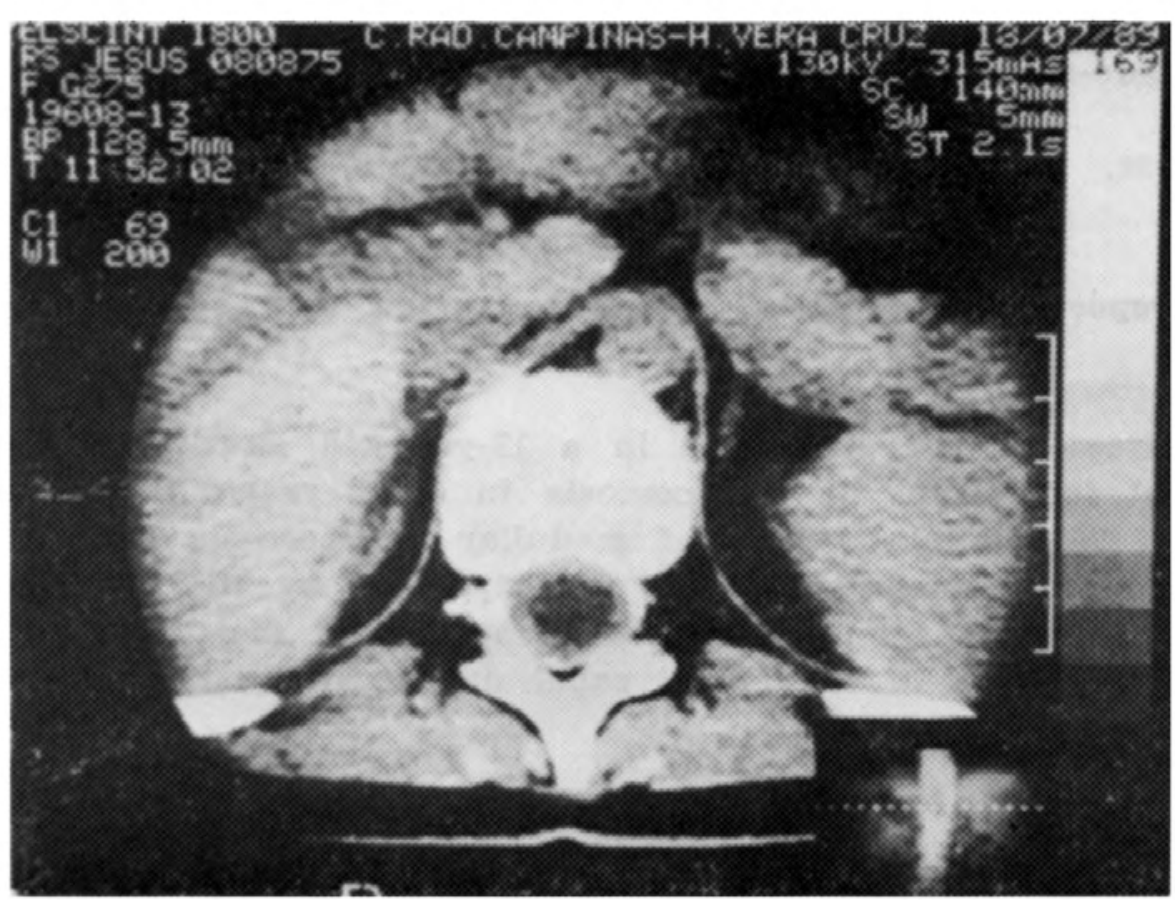

Fig. 1. Caso 1 (RSJ). A esquerda, CT de coluna mostrando lesão hipodensa intramedular central, comprometendo praticamente todo o diametro da medula a nivel T-11 e T-12. A direita, mielografia toraco-lombar mostrando bloqueio completo entre $T-11$ e T-12.

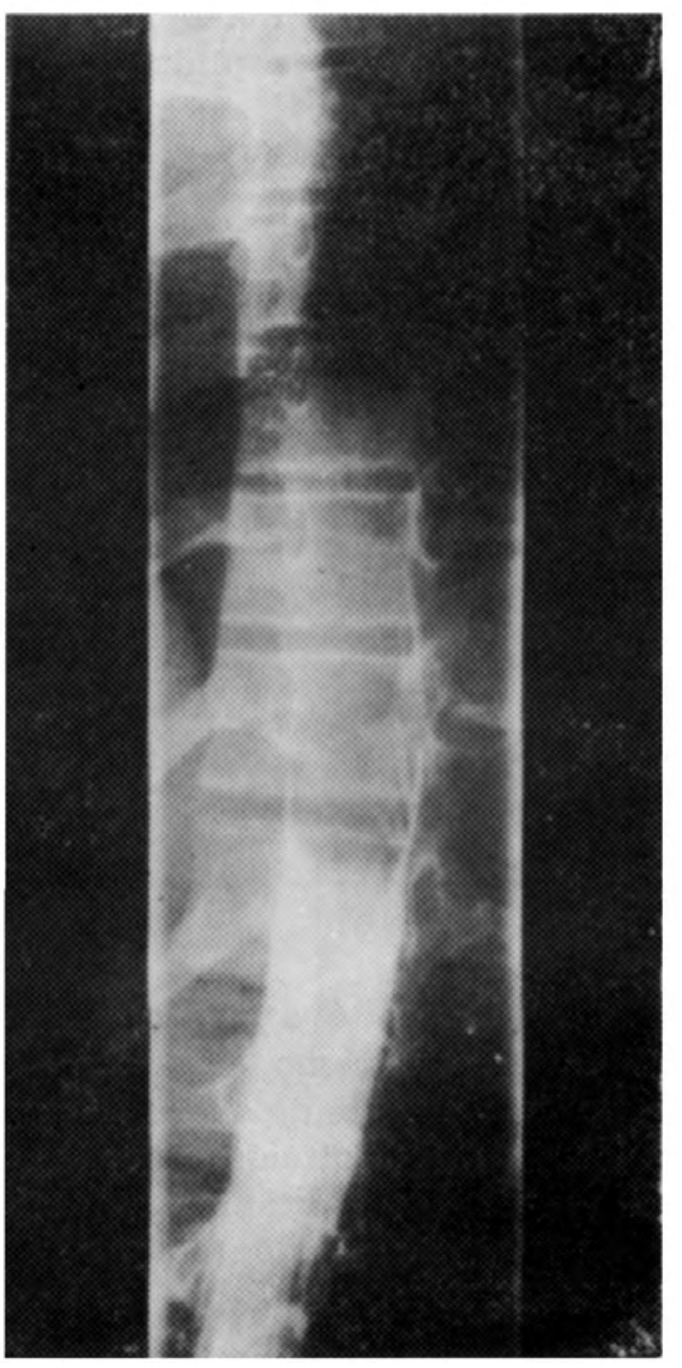

Caso 2 - EZFF, mulher de 51 anos de idade, procedente de zona rural, admitida em junho-1387 na Santa Casa de Vaiinhos, queixando-se há dois meses de cefaléia matinal e dores mal definidas em membros inferiores, acompanhadas de paresia progressiva. Na semana anterior, referia episódios de perda involuntária de urina. Evoluiu com piora da paraparesia, dificultando a deambulação. Ao exame apresentava-se afebril, normotensa e em boas condições gerais. Evidenciava-se paraparesia crural espástica, hiperreflexia profunda em membros inferiores e reflexo cutâneo-plantar em extensão, bilateralmente. Sensibilidade termo-álgica diminuída, simetricamente, a partir de LI. Exame de fundo de olho, papiledema bilateral. Nervos cranianos e de membros superiores sem alterações ao exame. Exames laboratoriais - Hemograma com leucocitose e desvio à esquerda, sem eosinofilia. Estudo radiológico simples: de coluna, normal; de crânio, com destruição da parte posterior da sela túrcica e das clinóides posteriores. Submetida então a TC de crânio, que mostrou dilatação importante de todo o sistema ventricular, porem com pouco edema transependimário. Realizada derivação ventrículo-peritoneal em 25- 
Tabela 1. Cisticercose intramedular: casos publicados.

\begin{tabular}{|c|c|c|c|c|c|c|c|}
\hline Autor & Ano & País & Idade & Sexo & Cerebr* & Diagnóstico & Nivel \\
\hline Rokitansky & 1856 & $?$ & $?$ & $?$ & $?$ & $?$ & $?$ \\
\hline Walton & 1881 & $?$ & $?$ & $?$ & + & $?$ & C3-C4 \\
\hline Pichler & 1900 & $?$ & $?$ & $?$ & $?$ & $?$ & T11, L1 \\
\hline Walibraun & 1917 & $?$ & $?$ & $?$ & + & $?$ & cerv. baixo \\
\hline Knapp & 1919 & EUA & 25 & $\mathbf{M}$ & - & Cirurgia & T4-T5 \\
\hline Salles & 1934 & Brasil & 12 & $\mathbf{M}$ & + & Necrópsia & médio-toracico \\
\hline Lopez & 1936 & Espanha & $?$ & $?$ & $\because$ & $?$ & $?$ \\
\hline Barini & 1954 & Brasil & 56 & $\mathbf{M}$ & - & Cirurgia & T10-T11 \\
\hline Costia & 1957 & Brasil & 40 & $\mathbf{M}$ & - & Cirurgia & $\mathrm{C} 7$ \\
\hline Trelles & 1958 & Peru & 32 & $\mathbf{F}$ & + & Necrópsia & C3-C4-L3-L4 \\
\hline Rocca & 1959 & Peru & 39 & $\mathbf{M}$ & + & Cirurgia & $\mathrm{T} 7$ \\
\hline \multirow[t]{2}{*}{ Cabieses } & 1959 & Peru & 42 & $\mathbf{M}$ & - & Cirurgia & T4 \\
\hline & & & 58 & $\mathbf{M}$ & + & Cirurgia & C5-C6 \\
\hline Dixon & 1961 & fndia & $?$ & $?$ & $?$ & $?$ & Cervical \\
\hline Figueiredo & 1963 & Brasil & 20 & $\mathbf{M}$ & - & Cirurgia & T12 \\
\hline Granés & 1963 & Brasil & 28 & $\mathbf{M}$ & - & Cirurgia & T6 \\
\hline Portugal & 1964 & Brasil & 40 & $\mathbf{M}$ & - & Cirurgia & C6-C7 \\
\hline Hesketh & 1965 & Singapura & 22 & $\mathbf{M}$ & - & Cirurgia & T4 \\
\hline Testa & 1965 & Itália & 40 & $\mathbf{M}$ & - & Cirurgia & $\mathrm{T} 8$ \\
\hline Singh & 1966 & fndia & 40 & $\mathbf{M}$ & + & Cirurgia & T7-T8 \\
\hline Arseni & 1967 & Romênia & $?$ & $?$ & $?$ & $?$ & T11-L1 \\
\hline Trelles & 1968 & Peru & 31 & $\mathbf{M}$ & - & Cirurgia & T9 \\
\hline Mehta & 1971 & India & 23 & $\mathbf{M}$ & - & Cirurgia & T11 \\
\hline Antoniuk & 1974 & Brasil & $?$ & $\mathbf{F}$ & $?$ & $?$ & T10 \\
\hline Queiroz & 1975 & Brasil & 60 & $\mathbf{F}$ & + & Necrópsia & T8 \\
\hline Mercado & 1976 & México & 22 & $\mathbf{F}$ & - & Cirurgia & T12 a L1 \\
\hline Natarajan & 1978 & fndia & 22 & $\mathbf{M}$ & - & Cimurgia & $\mathbf{T 4}$ \\
\hline Akiguchi & 1979 & Japão & 22 & $\mathbf{M}$ & - & Cirurgia & T8 a L2 \\
\hline Holtzman & 1986 & EUA & 18 & $\mathbf{M}$ & - & Cirurgia & $\mathbf{T 4}$ \\
\hline Sharma & 1987 & fndia & 42 & $\mathbf{M}$ & - & Cirurgia & T5 \\
\hline \multirow[t]{2}{*}{ Bally } & 1989 & Zimbabue & 20 & $\mathbf{M}$ & - & Cirurgia & $\mathbf{T 1}$ \\
\hline & & & 23 & $\mathbf{F}$ & - & Cirurgia & Cerv. baixo \\
\hline caso 1 & 1991 & Brasil & 13 & $\mathbf{F}$ & - & Cirurgia & T11-T12 \\
\hline
\end{tabular}

* cocxistência de sítio cerebral.

novembro-87. No pós-operatório, o líquido cefalorraquidiano lombar mostrou-se límpido e incolor, com prova de bloqueio negativa $(5,2$ hemácias e 4,0 leucócitos/mm3; SO mg/dL de proteínas; $81 \mathrm{mg} / \mathrm{dL}$, de glicose) e com reação de fixiação de complemento para cisticercose negativa. Na evolução, foi submetida a mielografia lombar, duas mielotomografias e uma tomografia simples, observando-se nos exames contrastados diversas vesículas provocando falhas de enchimento entre Ll e U (Fig. 2). Em 30-novembro-87 foi realizada laminectomia bilateral L2L4. Após incisão na dura-mater e aracnóide, houve saída de varitas vesículas (algumas espontaneamente) de pequeno diâmetro $(12$ no total, entre 0,5 e $1,0 \mathrm{~cm})$. Introduzido catéter de Nelaton no espaço subaracnóide acima e abaixo da incisão e injetado soro fisiológico, com saída de algumas vesículas; entretanto, sua» progressão era bloqueada acima de T12. O exame anátomo-patológico comprovou tratarem-se de cisticercos. A paciente evoluiu sem cefaléia e com melhora da paraparesia, voltando a deambular, havendo regressão das alterações de sensibilidade e esfincterianas. Perdeu-se seu seguimento após um ano. 
Tabela 5. Cisticercose medular subaracnóide: casos publicados.

\begin{tabular}{|c|c|c|c|c|c|c|c|}
\hline Autor & Ano & País & Idade & Sexo & Cerebr* & Diagnóstico & Nivel \\
\hline Westphal & 1865 & $?$ & $?$ & $?$ & $?$ & $?$ & Cauda equina \\
\hline Richter & 1891 & $?$ & $?$ & $?$ & + & Necrópsia & $\mathrm{C} 4-\mathrm{T} 10$ \\
\hline Minor & 1899 & $?$ & $?$ & $?$ & + & Necrópsia & Toracica \\
\hline Hartman & 1902 & Alemanha & $?$ & $?$ & $?$ & Punção LCR & - \\
\hline \multirow[t]{2}{*}{ Wollemberg } & 1905 & $?$ & $?$ & $?$ & + & Necrópsia & Cerv/lombar \\
\hline & & $?$ & $?$ & $?$ & + & Necrópsia & Filum term. \\
\hline Sterz & 1910 & Alemianha & $?$ & $?$ & $?$ & Punção LCR & - \\
\hline Vasiliu & 1921 & Itália & $?$ & $?$ & $?$ & $?$ & $?$ \\
\hline Redalie & 1921 & França & $?$ & $?$ & $?$ & $?$ & Cerv/torac. \\
\hline Castex & 1926 & Argentina & 25 & $F$ & + & Punção LCR & 一 \\
\hline Verga & 1926 & Itália & $?$ & $?$ & + & Necrópsia & $?$ \\
\hline Morawieka & 1927 & $?$ & $?$ & $?$ & + & Punção LCR & Cauda equina \\
\hline Bertrand & 1945 & França & $?$ & $?$ & $?$ & $?$ & Lombar \\
\hline Loyo & 1955 & México & $?$ & $?$ & $?$ & $?$ & Cauda equina \\
\hline Pennybaker & 1956 & $?$ & $?$ & $?$ & + & Cirurgia & Cervical \\
\hline Fracassi & 1956 & Argentina & 49 & $\mathbf{M}$ & $-i-$ & Punção LCR & - \\
\hline \multirow[t]{2}{*}{ De La Riva } & 1957 & Espanha & $?$ & $?$ & $t$ & Necrópsia & Lombossacra \\
\hline & & & $?$ & $?$ & + & Punção LCR & Lombossacra \\
\hline Rocca & 1959 & \multirow{2}{*}{\multicolumn{2}{|c|}{ Peru (15 casos) }} & & & & 13 cervicais \\
\hline & & & & & & & $\begin{array}{l}1 \text { dorsal } \\
1 \text { lombar }\end{array}$ \\
\hline Cabieses & 1959 & Peru & 12 & $\mathbf{M}$ & - & Cirurgia & $\mathrm{T} 3$ \\
\hline Cruz & 1961 & Brasil & 42 & $\mathbf{M}$ & - & Cirurgia & T11-L2-, L2-L3 \\
\hline \multirow[t]{2}{*}{ Calzado } & 1960 & México & $?$ & $?$ & $?$ & $?$ & Toracica \\
\hline & & & 26 & $\mathbf{M}$ & - & Cirurgia & $\mathrm{T} 6-\mathrm{T} 9$ \\
\hline \multirow[t]{3}{*}{ Canelas } & 1963 & Brasil & 32 & $\mathbf{F}$ & + & Cirurgia & T11-L2 \\
\hline & & & 42 & $\mathbf{M}$ & + & Cirurgia & T12-L2 \\
\hline & & & 27 & $\mathbf{M}$ & + & Cirurgia & T5-T9 \\
\hline Staimle & 1964 & México & 57 & $\mathbf{M}$ & $\rightarrow$ & Cirurgia & Cauda equina \\
\hline \multirow[t]{4}{*}{ Absalon } & 1965 & México & 45 & $\mathbf{M}$ & - & Cirurgia & T8-T9 \\
\hline & & & 35 & $\mathbf{M}$ & - & Cirurgia & $\mathrm{C} 5-\mathrm{C} 7$ \\
\hline & & & 63 & $\mathbf{M}$ & - & Cirurgia & $\mathrm{C} 2-\mathrm{C} 4$ \\
\hline & & & 40 & $\mathbf{M}$ & + & Cirurgia & L1-L2 \\
\hline Alanis & 1967 & México & 57 & $\mathbf{F}$ & $?$ & $?$ & L4-L5 \\
\hline \multirow[t]{3}{*}{ Trelles } & 1968 & Peru & 56 & $\mathbf{M}$ & + & Necrópsia & $\mathrm{C} 4$ \\
\hline & & & 46 & $\mathbf{M}$ & + & Necrópsia & $\mathrm{C} 4$ \\
\hline & & & 58 & $\mathrm{~F}$ & + & Necrópsia & cone/cauda \\
\hline Castano & 1969 & Colombia & 34 & $F$ & - & Cirurgia & Caudia equina \\
\hline Carmalt & 1975 & EUA & 24 & $\mathbf{M}$ & - & Cirurgia & Cauda equina \\
\hline Roy & 1976 & fndia & 40 & $\mathbf{M}$ & + & Cirurgia & Cone medular \\
\hline Minoli & 1985 & Itália & 60 & $\mathbf{F}$ & - & Mielografia & Toracolombar \\
\hline Kin & 1985 & EUA & 41 & $F$ & + & Mielografia & T12-L5 \\
\hline Salvoiardo & 1986 & Itália & 56 & $\mathbf{F}$ & - & Cirurgia & L1-L2 \\
\hline \multirow[t]{2}{*}{ Parter } & 1988 & França & 35 & $\mathbf{M}$ & - & Cirurgia & Cauda equina \\
\hline & & & 46 & $\mathbf{F}$ & - & Cirurgia & Cauda equina \\
\hline \multirow[t]{2}{*}{ Sperlescu } & 1989 & Brasil & 28 & $\mathbf{F}$ & + & Cirurgia & T12-L2 \\
\hline & & & 54 & $F$ & - & Cirurgia & L3-L5 \\
\hline \multirow{2}{*}{$\begin{array}{l}\text { Rossitti } \\
\text { casc } 2\end{array}$} & 1990 & Brasil & 34 & $\mathbf{F}$ & - & Cirurgia & LA-L5 \\
\hline & 1991 & Brasil & 51 & $\mathbf{F}$ & + & Cirurgia & L1-L4 \\
\hline
\end{tabular}

* coexistência de sítio cerebral. 


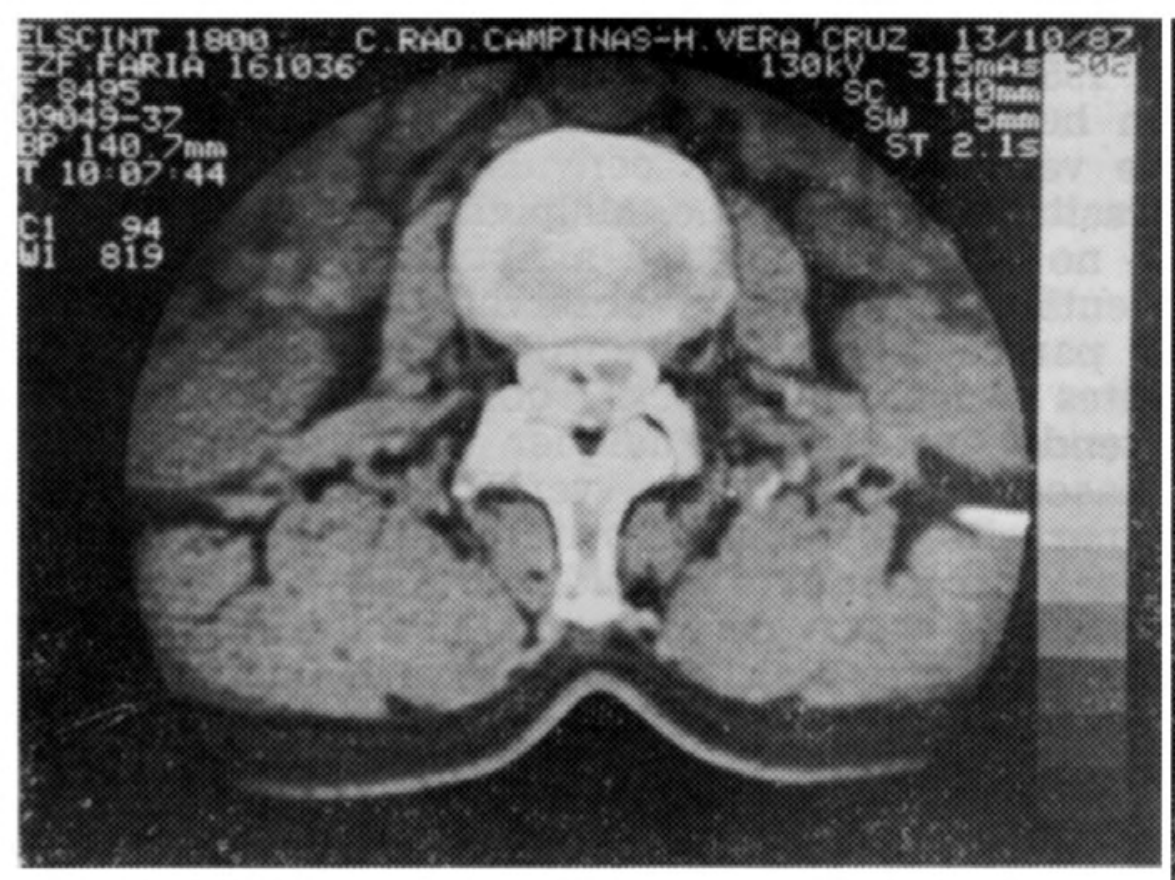

Fig. 2. Caso \& (EZFF). A esquerda, CT contrastada de coluna mostrando diversas vesiculas provocando falhas de enchimento entre $L-1$ e L-4. A direita, mielografia toraco-lombar mostrando falhas de enchimento entre L-1 e L-4 pela presença dos cisticercos.

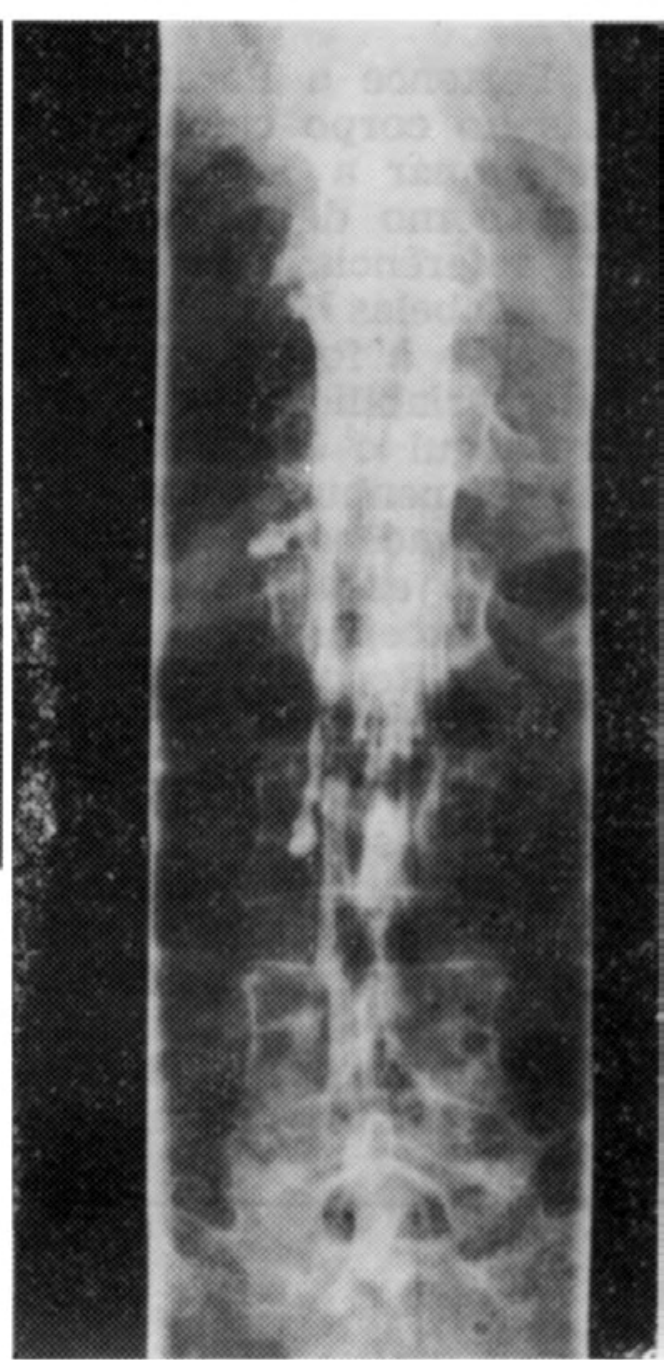

Tabela 3. Classificação anatômica, incidência e frequências relativas.

\begin{tabular}{ccc}
\hline Localização medular & No de Casos & $\%$ \\
\hline $\begin{array}{l}\text { Intramedular } \\
\text { Extramedular } \\
\text { leptomeníngea } \\
\text { epidural }\end{array}$ & 33 & 34,02 \\
& 61 & 62,88 \\
Total & 3 & 3,09 \\
\hline
\end{tabular}

Tabela 4. Distribuição anatômica e frequências relativas (\%).

\begin{tabular}{llr}
\hline & Nivel & No de Lesões \\
\hline Intramedular & Cervical & 8 \\
& Torácica & 23 \\
Leptomeníngea & Lombar & 21 \\
& Cervical & 16 \\
& Torácica & 19 \\
& Lombar & 12 \\
\hline
\end{tabular}




\section{COMENTARIOS}

Pertence a Paranoli, em 1550, a primeira descrição de um caso com vesículas no corpo caloso de um homem e atribui-se a Paracelso (1650) o mérito de relacionar a coexistência de vesículas e crises convulsivas, sendo elas identificadas como de natureza parasitária por Ridi e Malpighi, em 1686 ${ }^{\prime}$ io. A $_{\text {A }}$ primeira referência a cisticercose no canal raquídeo é atribuída a Rokitansky, em 1856» (Tabelas 1 e 2). Desde então, a frequência observada da forma medular, em relação à forma encefálica, parece estar em torno de $58 \%$. n. No entanto, há grande variabilidade em diferentes series, com Rocca ${ }^{21}$ observando taxa de 19,7\%, Takayanagui e Jardim 26 ão tendo encontrado qualquer um entre 500 casos, e Canelas 6, nenhum, entre 276 casos. Posteriormente, verificou-se a existência de três localizações possíveis do parasito entre as estruturas medulares: leptomeníngea (no espaço subaracnóide), intramedular e epidural. Esta última é considerada excepcional, com apenas três casos descritos na literatura, por: Busse, 19311.10,20, Rocca. 195921 e, recentemente Vlok em 198830 , mencionou um caso com vários cisticercos no espaço epidural e no próprio corpo vertebral, levando a destruição óssea (Tabela 3).

Estudos anteriores estimavam frequência 6 a 8 vezes maior da localização leptomeníngea em relação à intramedular $n>24$, porém, numa ampla revisão da literatura, encontramos 95 casos publicados da forma espinhal, somados a dois casos de nossa casuística, num total de 97 casos, dentre os quais 61 com cistos subaracnóides e 33, intramedulares. Portanto, 62,88\% dos casos apresentam cistos nas leptomeninges e $34,02 \%$ intramedulares, proporcionando frequência apenas 1,84 vezes maior. (Tabela 3 ).

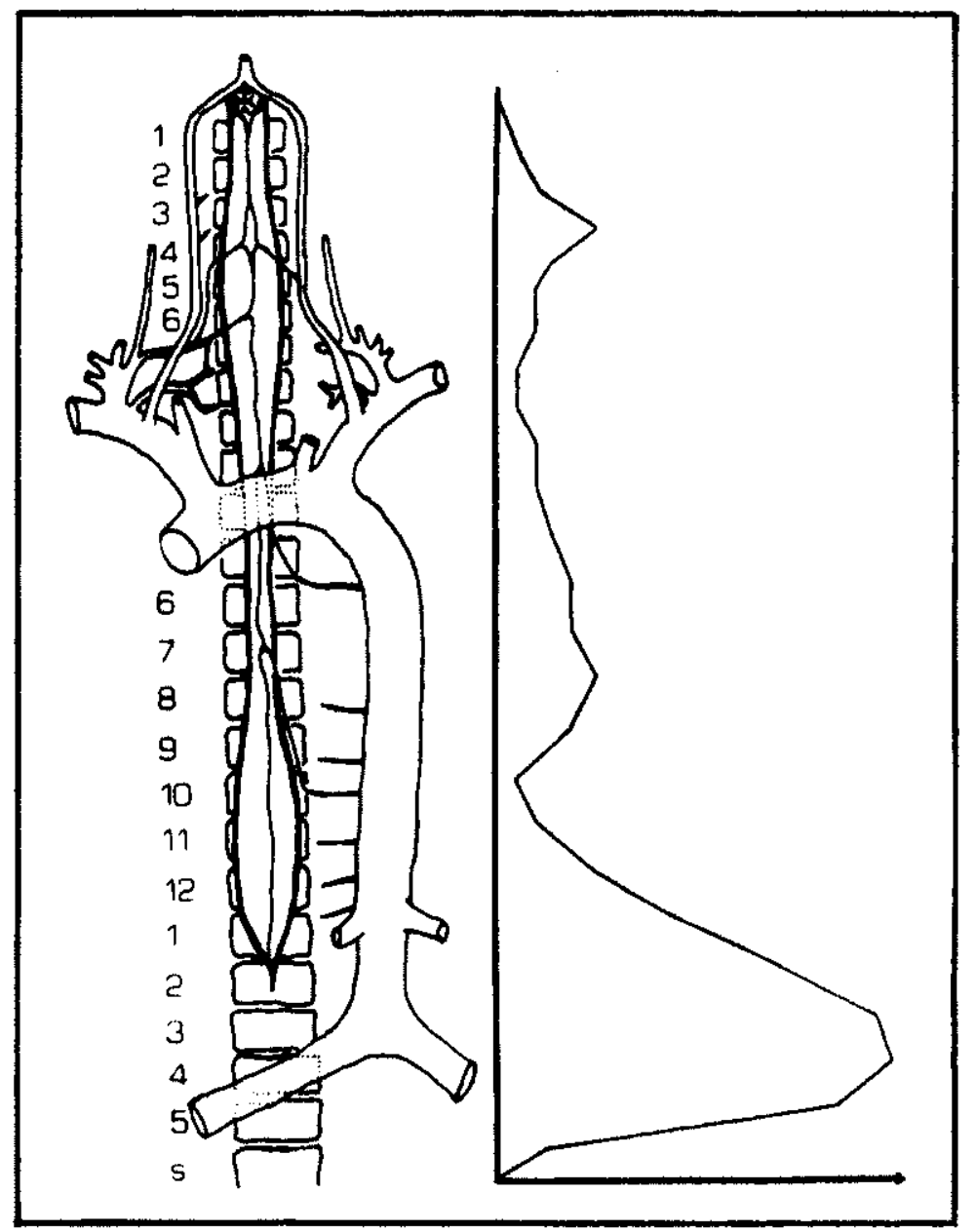

Fig. 3. Representação esquematica da medula no interior da coluna vertebral (à esquerda) comparauda ao gráfico da distribuiça a dos cisticercos no espaço subanacnóde da medula (à direita). $V \ell-s e$, que na cisticercose medular subaracnoide, os cisticercos são mais frequentemente encontrados onde o espaço perimedular é mais amplo.

Dentro da revisão feita encontramos: faixa etária entre 12 a 63 anos, com média de 26 anos; proporção de 1 mulher para 2 homens com cisticercose medular; $33,3 \%$ dos casos intramedulares e 59,4\% dos leptomeníngeos apresentavam evidência de coexistirem cistos encefálicos. Clinicamente, a maioria dos casos parece apresentar quadro piramidal deficitário e de liberação em membros inferiores, dores muitas vezes radiculares e obedecendo a distribuição dermatomérica, por vezes associadas a déficits sensitivos variados e, ainda, distúrbios esfincterianos e impotência sexual. Essas manifestações clínicas seriam resultantes do 
efeito de massa causado pela presença do cisto, da reação inflamatória que ocorre ao seu redor e da degeneração da medula devida a insuficiência vascular n.14,24. A patogenia da cisticercose medular ainda não é bem definida. Segundo Queiroz et al. ii, a disseminação arterial é a mais provável via de acesso do parasito ao parênquima medular. Admitiram essa possibilidade, baseados na distribuição topográfica dos cisticercos verificada em sua revisão da literatura (1975), em que encontraram 16 casos somados a 1 de sua experiência, dentre os quais 2 apresentavam lesões em diferentes níveis de medula. Encontraram 19 lesões assim distribuídas: 5 cervicais, 12 torácicas e 2 lombares. Essa distribuição é confirmada em nossa revisão com 33 lesões, sendo 8 cervicais, 23 torácicas e 2 lombares (Tabela 4). Tal distribuição é estatisticamente proporcional ao fluxo sanguíneo de cada nível medular, direcionando os dados a favor da hipótese da via hematogênica. Nessa mesma análise, Queiroz et al.n coloca a falta de evidências para a via ventrículo-ependimária, já que se o parasito descesse pelo canal central da medula, seria mais provável que a maioria das vesículas fosse encontrada a nível cervical e, não, predominantemente na região torácica média como ocorre na realidade.

Na forma extramedular leptomeníngea foram já relatadas opiniões segundo as quais o embrião invadiria o espaço subaracnóide espinhal, através das conexões do plexo venoso vertebral interno, e que a cisticercose racemosa das cisternas da base seria secundária ao acometimento espinhal primário, de onde os cisticercos poderiam ascender ao espaço subaracnóideo intracraniano, através do forame magno 25 .

Como observado nesta revisão, a forma de cisticercose leptomeníngea pura é rara e, assim, consideramos mais plausível a hipótese defendida por outros autores 7. n,2i,2S, ${ }_{\text {e }}$ i a qual a migração das larvas ocorre do espaço subaracnóideo encefálico para o espinhal, sendo a maioria das larvas encontradas nas porções superiores da medula (Tabela ${ }^{4}$ ) . Acrescentamos, neste estudo, uma justificativa anatômica para este raciocínio, apresentada a seguir. A medula, que tem aproximadamente $45 \mathrm{~cm}$ no homem e $41 \mathrm{~cm}$ na mulher, pode ser dividida em: uma porção superior, que mede cerca de $2 \mathrm{~cm}$ de comprimento' e se estende do limite com o bulbo até a intumescência cervical, indo do forame magno até a terceira vértebra cervical; uma intumescência cervical, que se estende da terceira vértebra cervical à segunda vértebra torácica, com extensão de 10 a $12 \mathrm{~cm}$ e com seu maior diâmetro correspondente à sexta vértebra cervical $(14 \mathrm{~mm})$; uma região intermédia estendendo-se da segunda vértebra torácica à nona ou décima vértebra torácica, medindo cerca de 18 a $22 \mathrm{~cm}$; uma intumescência lombar, que se estende da nona ou décima vértebra torácica à primeira ou segunda vértebra lombar, medindo 7 a $9 \mathrm{~cm}$ de comprimento e tendo diâmetro menor que a intumescência cervical, alcançando $12 \mathrm{~mm}$ de diâmetro na décima segunda vértebra torácica; um cone terminal; e um filamento terminal 27.

O calibre da medula muda consideravelmente, tendo uma circunferência de $38 \mathrm{~mm}$ na intumescência cervical, $33 \mathrm{~mm}$ na lombar e $27 \mathrm{~mm}$ na porção intermédia, com diâmetros transversal e ántero-posterior respectivamente de $13 \mathrm{~mm}$. e $9 \mathrm{~mm}$ na intumescência cervical, $12 \mathrm{~mm}$ e $9 \mathrm{~mm}$ na intumescência lombar, $10 \mathrm{~mm}$ e $8 \mathrm{~mm}$ na porção intermédia 27 . Além disso, a medula, em qualquer ponto que se examine, ocupa sempre o centro do canal vertebral, que também apresenta variações (maior e com formato triangular, nas regiões cervical e lombar; menor e arredondado, na região torácica) 27.

$O$ espaço perimedular, situado entre a medula e a parede óssea da vértebra, é bastante variável ( 3 a $8 \mathrm{~mm}$ ), sendo maior nas regiões situadas imediatamente acima das intumescências cervical e lombar 27, correspondendo às porcões onde os cisticercos são mais frequentemente encontrados no espaço subaracnóideo (Fig. 3). Portanto, parece-nos que as larvas migram do espaço subaracnóideo encefálico para o espinhal, localizando-se nessas regiões onde o espaço perimedular é mais amplo.

Por outro lado, a cisticercose medular deve ser considerada no diagnóstico diferencial de mielopatias compressivas, particularmente em áreas endêmicas.

Agradecimentos - Os autores desejam expressar seus agradecimentos ao Prof. Dr. Luciano de Souza Queiroz (Departamento de Anatomia Patológica da FCM-UNICAMP) e a Profa. Dra. Vilma Clóris de Carvalho (Departamento de Anatomia da FCM-UNICAMP) pelos incentivos na realização deste estudo. 


\section{REFERÊNCIAS}

1. Akiguchi M, Fujiwara T, Matsuyama H, Muranaka H, Kameyama M. Intramedullary spinal cysticercosis. Neurology 1979, 29:1531-1534.

2. Antoniuk AM, Moro, Perez W. Cisticerco intramedular único (Resumo). VI Congresso Brasileiro de Neurologia. Rio de Janeiro, 1974, p 114.

3. Bally GG. Unusual cystic lesions oi: the spinal cord. J Roy Soc Med 1989, 103:1227-1228.

4. Barini $O$. Cisticerco macrocistico intramedular: extirpação cirúrgica. Arq Neuro-Psiquiat (São Paulo) 1954, 12-264-266.

5. Briceño CE, Biagi F, Martinez B. Cisticercosis: observaciones sobre 97 casos de autopsia. Prensa Med Mex 1961, 26:193-197.

6. Canelas HM. Neurocisticercose: incidência, diagnóstico e formas clínicas. Arq. NeuroPsiquiat (São Paulo) 1962, 20:1-16.

7. Canelas HM, Cruz OR, Escalante OAD. Cysticercosis of the nervous system: less frequent clinical forms. III. Spinal cord forms. Arq Neuro-Psiquiatr (São Paulo) 1963, 21:77-86.

8. Carmalt JE, Theis J, Goldstein E. Spinal cysticercosis West J Med 1975, 123:311-314.

9. Carvalho-Filho P. Neurocisticercose. Medicina em Monografias. Programa Nacional de atualização Médica Fontoura-Wyeth 1987, Vol. 3, n? ö.

10. Costa AL, Figueiredo HB, Serra JP. Cisticercose do sistema nervoso. Arq Bras Med 1957, 47:407-430.

11. De Souza Queiroz L, Pellegrini A Filho, Callegaro D, De Faria LL. Intramedullary cysticercosis: case report, literature review and comments on pathogenesis. J Neurol Sei 1975, 26:61-70.

12. Guccione A. La Cisticercosi del Sistema Nervoso Centrale Umano. Milano: Soc Edit Libraria, 1919.

13. Hellmeister CR, Faria JL. Neurocisticercose: dados neeroscópicos. Rev Ass Med Bras 1973, 19:281-282.

14. Holtzman RN, Hughes JEO, Sachdev RK, Jarenwattananon A. Intramedullary cysticercosis. Surg Neurol 1986, 26:187-191.

15. Kim KS, Weinberg PE. Spinal cysticercosis, Surg Neurol 1985, 24:80-82.

16. Kimpton AR, Cysticercus racemosus (Taenia solium) infection of spinal cord: with report of case. Surg Gynec Obstet 1920, 30:198-200.

17. Minguetti G, Ferreira MVC. Ação de corticóides na fase aguda da neurocisticercose: nota preliminar. Arq Neuro-Psiquiat 1982, 40:77-85.

18. Minoli L, Semaldi G, Malfitano $M$, Marone $P$, Bussone $G$. Spinal cysticercosis: a case report and its treatment with praziquantel (Letter). Boll 1st Sieroter Milan 1985, 64:343-344.

19. Parter F. Racemous cysticercosis of the cauda equina and cystic aracnoidites : a propos of 2 cases. Neurochirurgie 1988, 34:2805.

20. Portugal JR, Oliveira C. Cisticercose intramedular. J Bras Neurol 1964, 16:3-12.

21. Rocca ED. Cisticercosis intramedular. Rev. Neuropsiquiat (Lima) 1959, 22:166-173.

22. Rossitti SL, Roth-Vargas AA. Cisticercose espinhal leptomeníngea pura. Arq Neuro-Psiquiat (São Paulo) 1990, 48:366-370.

23. Savoiardo M, Cimino C, Passerini A, La. Mantia L. Mobile myélographie filling defects: spinal cysticercosis. Neuroradiology 1986, 28:166-169.

24. Sharma BS, Banerjee AK, Kak VK. Intramedullary spinal cysticercosis: case report and review of literature. Chin Neurol Neurosurg 1987, 89:111-116.

25. Sperlescu A, Balbo RJ, Rossitti SL. Breve comentário sobre a patogenia da cisticercose espinhal. Arq Neuro-Psiquiatr (São Paulo), 1989, 47:105-109.

26. Takayanagui OM, Jardim E. Aspectos clínicos da neuroeisticercose : análise de 500 casos. Arq Neuro-Psiquiat (São Paulo) 1983, 41:50-63.

27. Testut L, Latarjet A. Anatomia Humana. Barcelona: Salvat, 1979, Vol. 2.

28. Trelles JO, Caceres A, Palomino L. La Cysticercose médullaire. Rev Neurol (Paris) 1970, 123:187-202.

30. Vloki G J. Vertebral cysticercosis: a case report. S Afr Med J 1988, 73:730-731. 